\title{
Thermal correlators in the hadron resonance gas: a dual Hagedorn distance* **
}

\author{
E. Megías ${ }^{a}, b$, E. Ruiz Arriola ${ }^{a}$, L.L. SAlcedo ${ }^{a}$ \\ ${ }^{a}$ Departamento de Física Atómica, Molecular y Nuclear and Instituto Carlos I de \\ Física Teórica y Computacional, Universidad de Granada, \\ Avenida de Fuente Nueva s/n, 18071 Granada, Spain \\ ${ }^{b}$ Departamento de Física Teórica, Universidad del País Vasco UPV/EHU, \\ Apartado 644, 48080 Bilbao, Spain
}

Fluctuations and correlations of conserved quantities in the confined phase of QCD are a viable way to characterize the existence of exotic and missing states with given quantum numbers in the hadronic spectrum. We study a realization of the Hadron Resonance Gas model in the light quark (uds) flavor sector of QCD to study the fluctuations and static correlators of electric charge, baryon number and strangeness. It is also conjectured an interesting duality between the correlators at zero temperature, and the fluctuations of integrated quantities at low temperatures, leading to the appearance of a dual Hagedorn distance for the former.

PACS numbers: 11.10.Wx, 12.38.-t, 12.38.Lg

\section{Introduction}

A fundamental quantity for the study of the thermodynamics of QCD is the partition function, which writes

$$
Z_{\mathrm{QCD}}=\operatorname{Tr} e^{-H_{\mathrm{QCD}} / T}=\sum_{n} e^{-E_{n} / T}
$$

where $E_{n}$ are the eigenvalues of the QCD Hamiltonian. This illustrates the connection between the thermodynamics and the spectrum of QCD. An

* Talk by E. Megías at "Excited QCD 2018", Kopaonik, Serbia, March 11-15, 2018.

** Work supported by the Spanish MINEICO and European FEDER funds (grants FIS2014-59386-P, FIS2017-85053-C2-1-P and FPA2015-64041-C2-1-P), Junta de Andalucía (grant FQM-225) and Basque Government (grant IT979-16). The research of E.M. is also supported by the Ramón y Cajal Program of the Spanish MINEICO, and by the Universidad del País Vasco UPV/EHU, Bilbao, Spain, as a Visiting Professor. 
explicit realization in the confined phase is given by the Hadron Resonance Gas (HRG) model [1] where the hadronic states compiled by the PDG [2] are considered as stable, non-interacting and point-like particles. Microscopic models have also been used to compute the meson and baryon spectra, such as e.g. the Relativized Quark Model (RQM) [3, 4]. In general, these models predict more states than those reported by the PDG, leading to the idea of missing states in the QCD spectrum. Moreover, apart from the conventional mesons and baryons, it has been conjectured the possible existence of exotic states, i.e. those with exotic quantum numbers like tetraquarks, pentaquarks or hybrid states, all of them forming color neutral states. Our recent studies on the Polyakov loop and the corresponding Entropy shift due to a heavy quark, suggest that conventional hadrons from the PDG or RQM are not enough to saturate the sum rules, and there are in the spectrum: i) conventional missing states $([Q \bar{q}]$ and $[Q q q])$, and ii) hybrid states $([Q \bar{q} g]$ and $[Q q q g])[5-7]$.

While the HRG approach has been very successful in the description of the equation of state (EoS) of the confined phase of QCD, leading to a good agreement with the lattice data for $T \lesssim 0.8 T_{c}$ (see e.g. Refs. [5,8] and cited references), above this regime the HRG assumptions are invalid and the approach breaks down. In fact, the partition function becomes divergent at some finite value of the temperature (the so-called Hagedorn temperature) after summation over a spectrum with an exponential growth of the density of states, i.e.

$$
Z_{\mathrm{HRG}}=\operatorname{Tr} e^{-H_{\mathrm{HRG}} / T} \underset{T \rightarrow T_{H}^{-}}{\longrightarrow} \frac{A}{T_{H}-T}, \quad T_{H} \approx 150 \mathrm{MeV}
$$

The EoS is sensitive to the spectrum of QCD as a whole, and it is interesting to study other thermal observables which allow to distinguish between different channels of the spectrum. In this contribution we will study the fluctuations and correlations of conserved charges, and present them as a tool to check the validity of the HRG approach, as well as to help in the characterization of exotic and missing states in several sectors of the spectrum of QCD.

\section{Fluctuations of Conserved Charges in a Thermal Medium}

Fluctuations of conserved charges $\left(\left[Q_{a}, H\right]=0\right)$ are a way of selecting quantum numbers $[9$. In the $(u d s)$ flavor sector the only conserved charges are the number of $u, d$ and $s$ quarks or equivalently the electric charge $Q$, the baryon number $B$, and the strangeness $S$. We will study in this section the HRG realization of the thermal fluctuations of these quantities. 
While in the hot vacuum (with no chemical potentials) the thermal expectation values of conserved charges are vanishing, i.e. $\left\langle Q_{a}\right\rangle_{T}=0$ where $Q_{a} \in\{Q, B, S\}$, they present statistical fluctuations, characterized by susceptibilities and defined as 10,11

$$
\chi_{a b}(T) \equiv \frac{1}{V T^{3}}\left\langle\Delta Q_{a} \Delta Q_{b}\right\rangle_{T}, \quad \Delta Q_{a}=Q_{a}-\left\langle Q_{a}\right\rangle_{T} .
$$

These quantities can also be computed as $\left.\chi_{a b}(T) \sim \frac{\partial^{2} \Omega}{\partial \mu_{a} \partial \mu_{b}}\right|_{\mu_{a, b}=0}$ from the grand-canonical partition function, given by

$$
Z=\operatorname{Tr} \exp \left[-\left(H-\sum_{a} \mu_{a} Q_{a}\right) / T\right], \quad \Omega=-T \log Z .
$$

Within the HRG model, the charges are carried by various species of hadrons, $Q_{a}=\sum_{i} q_{a}^{i} N_{i}$, where $q_{a}^{i} \in\left\{Q_{i}, B_{i}, S_{i}\right\}$ is the charge of the $i$ thhadron corresponding to symmetry $a$, and $N_{i}$ is the number of hadrons of type $i$. Hence, the susceptibilities are computed as [12,13]

$$
\chi_{a b}(T)=\frac{1}{V T^{3}} \sum_{i, j \in \text { Hadrons }} q_{a}^{i} q_{b}^{j}\left\langle\Delta N_{i} \Delta N_{j}\right\rangle_{T}, \quad a, b \in\{Q, B, S\} .
$$

The averaged number of hadrons is $\left\langle N_{i}\right\rangle_{T}=V \int \frac{d^{3} k}{(2 \pi)^{3}} \frac{g_{i}}{e^{E_{k, i} / T}-\xi_{i}}$, with $E_{k, i}=$ $\left(k^{2}+M_{i}^{2}\right)^{1 / 2}, g_{i}$ is the degeneracy and $\xi= \pm 1$ for bosons/fermions. Since the different species are uncorrelated, then $\left\langle\Delta n_{\alpha} \Delta n_{\beta}\right\rangle_{T}=\delta_{\alpha \beta}\left\langle n_{\alpha}\right\rangle_{T}(1+$ $\left.\xi_{\alpha}\left\langle n_{\alpha}\right\rangle_{T}\right)$ for the occupation numbers, where $\alpha$ and $\beta$ stand for any complete set of quantum numbers. Since $\left\langle n_{\alpha}\right\rangle_{T} \ll 1$, then $\left\langle\Delta Q_{a} \Delta Q_{b}\right\rangle_{T} \approx$ $\sum_{i \in \text { Hadrons }} q_{a}^{i} q_{b}^{i}\left\langle N_{i}\right\rangle_{T}$. The remarkable good agreement of the EoS found between PDG and RQM [5, 8] compared with lattice QCD, while still reasonable for $T \lesssim 150 \mathrm{MeV}$, gets a bit spoiled in terms of fluctuations leading to the conclusion that fluctuations may serve as a diagnostic tool to study missing states in the spectrum. For instance, the $B B$ susceptibility suggests that the RQM has too many baryonic states but not too many charged states [12,13]. Eventually at the Hagedorn temperature, $T_{H}$, the HRG susceptibilities diverge, $\left.\chi_{a b}\right|_{\mathrm{HRG}} \sim 1 /\left(T_{H}-T\right)$, overcoming the quark model values expected in the large temperature limit; for $u d s$ quarks one should get $\chi_{Q Q} \rightarrow \sum e_{q}^{2} \equiv 2 / 3, \chi_{B B} \rightarrow 1 / N_{c}$ and $\chi_{S S} \rightarrow 1$.

\section{Local Correlations in a Thermal Medium}

After the overall success of the HRG for the susceptibilities, it is tempting to extend the analysis at the local level. We are thus interested in the 
computation of the retarded correlators of conserved quantities

$$
C_{\mu \nu}^{a b}(x)=\left\langle j_{\mu}^{a}(x) j_{\nu}^{b}(0)\right\rangle .
$$

The static version of these correlators are related to the susceptibilities $\chi_{a b}(T)$ through

$$
\chi_{a b}(T)=\frac{1}{T^{3}} \int d^{3} x C_{00}^{a b}(0, \vec{x}) .
$$

In the case of spin $1 / 2$ particles the vector currents are defined as $j_{a}^{\mu}(x)=$ $\bar{\Psi}(x) \gamma^{\mu} \widehat{Q}_{a} \Psi(x)$ with $\widehat{Q}_{a}$ a matrix that specifies the charge. In this case the static part behaves at small distances as $\left\langle j_{0}^{a}(\vec{x}) j_{0}^{b}(0)\right\rangle \sim r^{-6}$ with $r=|\vec{x}|$. We can extend this result to finite temperature by using the Poisson's summation formula [14]. From a comparison of the zero and finite temperature correlators, one finds that the thermal corrections in the static correlator at small distances start at $\mathcal{O}\left(r^{-2}\right)$ in the case of spin $1 / 2$ particles.

Within the HRG model, we need to evaluate

$$
C_{\mu \nu}^{a b}(x)=\sum_{M \in \text { Mesons }} \frac{1}{2} g_{M} q_{M}^{a} q_{M}^{b} C_{\mu \nu}^{J_{M}}(x)+\sum_{B \in \text { Baryons }>0} g_{B} q_{B}^{a} q_{B}^{b} C_{\mu \nu}^{J_{B}}(x),
$$

where $C_{\mu \nu}^{J}(x) \equiv\left\langle j_{\mu}(x) j_{\nu}(0)\right\rangle_{J}$ are the correlators of free particles of spin $J$. The summations in $M$ and $B$ run over the spin multiplets of mesons and baryons, each of them with degeneracy $g_{M}=\left(2 J_{M}+1\right)$ and $g_{B}=\left(2 J_{B}+1\right)$ respectively. Baryons and antibaryons contribute to the correlators in the same amount, so that we have considered in $\sum_{B}$ a summation over baryons only, and multiplied it by a factor 2 . It is possible to extend the analysis to particles of any spin by using the Bargmann-Wigner formalism 15] and to obtain the correlators in Euclidean space (details will be provided elsewhere [16]). The small distance behavior of the static correlators, either at zero or finite temperature, reads (for $J>0$ and up to a factor)

$$
C_{00}^{a b J}(r) \underset{r \rightarrow 0}{\sim} \delta_{a b} \frac{m^{2}}{r^{4}} \frac{1}{(m r)^{4 J}} .
$$

As an example, the lowest lying states contributing to the summations of Eq. (8) are $M \in\left\{\pi^{+}, \pi^{-}, \pi^{0}\right\}$ and $B \in\{p, n\}$. We display in Fig. 1 the result of the static correlators at zero and finite temperature in the confined phase of QCD, within the HRG model. Notice the important growth of the zero temperature correlator at short distances. Unfortunately, to the best of our knowledge there are no lattice studies for these quantities yet. 

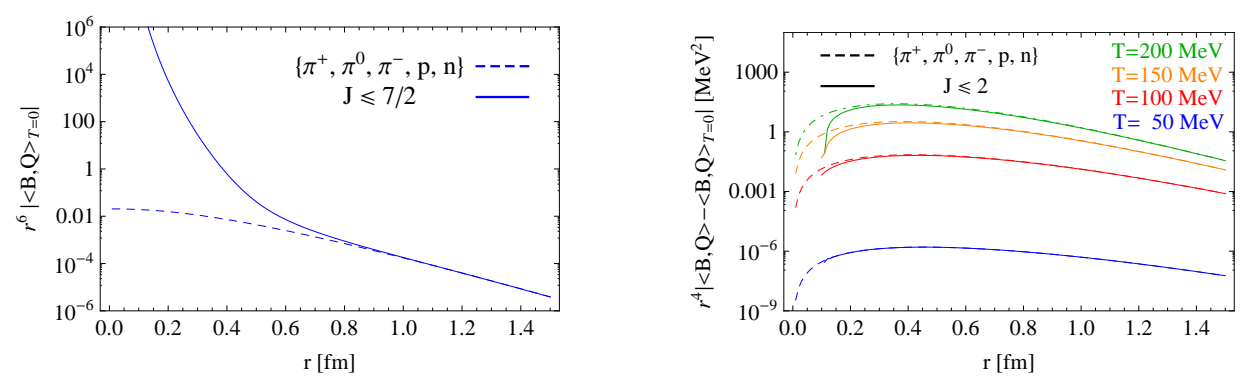

Fig. 1. Static correlator $C_{00}(0, \vec{x}) \equiv\langle B(\vec{x}) Q(0)\rangle$ at zero temperature (left panel), and finite temperature (right panel), including pions and nucleons (dashed lines), and hadrons with $J \leq 7 / 2$ and $J \leq 2$ from the RQM spectrum [3, 4] (solid lines).

\section{Dual Hagedorn distance in the correlators}

We may conjecture the existence of a formal analogy between the static correlators at zero temperature and the finite temperature susceptibilities. From the asymptotic behaviors of these two quantities,

$$
\left.C_{00}^{a b}(0, \vec{x})\right|_{T=0} \underset{r \rightarrow \infty}{\sim} e^{-2 m r} \quad \text { and } \quad \chi_{a b}(T) \underset{T \rightarrow 0}{\sim} e^{-m / T},
$$

where $m$ is the mass of the lowest-lying state, one concludes that they have a similar behavior after considering the replacement $r \leftrightarrow 1 / T$. In particular, the existence of a limiting temperature for the validity of the hadronic representation of $\chi_{a b}(T)$, i.e. $T<T_{H}$, cf. Eq. (2), might have its counterpart in the existence of a limiting distance in the hadronic representation of the correlators $\left(r>r_{H}\right)$. In fact, given the behavior of the static correlators for particles of spin $J$, cf. Eq. (9), we expect that after summation over hadrons of higher and higher spin, the static correlators within the HRG model present a divergence at some finite value of the distance

$$
C_{00}^{\mathrm{HRG}}(r)=\sum_{J} C_{00}^{J}(r) \underset{r \rightarrow r_{H}^{+}}{\longrightarrow} \infty
$$

When using $T_{H} \approx 150 \mathrm{MeV}$ one gets $r_{H} \simeq 1 /\left(2 \pi T_{H}\right) \approx 0.21 \mathrm{fm}$, where the factor $1 /(2 \pi)$ is standard in finite $T$ computations. Notice that this value of $r_{H}$ is of the same order of magnitude than the distance at which the growth in Fig. 1 (left) become significant, when including hadrons with $J \leq 7 / 2$. This would be analogous to the divergence of the partition function at the Hagedorn temperature, after summation over a spectrum with an exponential growth of the density of states. As in the susceptibilities case the divergence appears as a purely hadronic feature, but we find this to 
happen for smaller distances than $0.6 \mathrm{fm}[16]$ where the hadronic correlation overcomes the quark correlation which should dominate for $r \rightarrow 0$.

\section{Conclusions}

At very low temperatures hadrons can be considered as a complete basis of states in terms of a HRG model. However, close to the deconfinement crossover of QCD, many hadrons are needed to saturate the sum rules, so that this regime turns out to be very interesting for the characterization of missing states in the spectrum. In this work we have argued that fluctuations and correlations of conserved charges can be used to study the existence of missing and exotic states in three different sectors: i) electric charge, ii) baryon number, and iii) strangeness. Finally, we have conjectured a duality between zero temperature correlators and finite temperature susceptibilities. This duality leads to the appearance of a dual Hagedorn distance in the correlators. It would be desirable to confront these results for the correlators with future results on the lattice.

\section{REFERENCES}

[1] R. Hagedorn, Lect. Notes Phys. 221 (1985) 53-76.

[2] C. Patrignani, et al., Review of Particle Physics, Chin. Phys. C40 (10) (2016) 100001

[3] S. Godfrey, N. Isgur, Phys. Rev. D32 (1985) 189-231.

[4] N. Isgur, M.B. Wise, Phys. Lett. B232 (1989) 113.

[5] E. Ruiz Arriola, L.L. Salcedo, E. Megias, Acta Phys. Polon. B45 (12) (2014) $2407-2454$.

[6] E. Megias, E. Ruiz Arriola, L.L. Salcedo, Phys. Rev. Lett. 109 (2012) 151601.

[7] E. Megias, E. Ruiz Arriola, L.L. Salcedo, Phys. Rev. D94 (9) (2016) 096010.

[8] E. Megias, E. Ruiz Arriola, L.L. Salcedo, Nucl. Phys. Proc. Suppl. 234 (2013) 313-316.

[9] M. Asakawa, M. Kitazawa, Prog. Part. Nucl. Phys. 90 (2016) 299-342.

[10] A. Bazavov, et al., Phys. Rev. D86 (2012) 034509.

[11] R. Bellwied, S. Borsanyi, Z. Fodor, S.D. Katz, A. Pasztor, C. Ratti, K.K. Szabo, Phys. Rev. D92 (11) (2015) 114505.

[12] E. Ruiz Arriola, W. Broniowski, E. Megias, L.L. Salcedo, YSTAR2016 MiniProceedings (2016), 136-147, arXiv:1612.07091.

[13] E. Megias, E. Ruiz Arriola and L.L. Salcedo, PoS Hadron 2017, 232 (2018).

[14] E. Megias, E. Ruiz Arriola, L.L. Salcedo, Phys. Rev. D74 (2006) 065005.

[15] V. Bargmann, E.P. Wigner, Proc. Nat. Acad. Sci. 34 (1948) 211.

[16] E. Megias, E. Ruiz Arriola, L.L. Salcedo, work in progress (2018). 